\title{
Fatigue Life Prediction and Optimization of Pipeline Under Random Loads
}

\author{
Du Zhong-lei ${ }^{1,}$, , Liao Ri-dong ${ }^{1, b}$ \\ ${ }^{1}$ School of Mechanical Engineering Beijing Institute of Technology, Beijing 10081 \\ adzl140@bit.edu.cn, bliaord@bit.edu.cn
}

Keywords: Pipeline, Power spectrum density, Random vibration, Fatigue

\begin{abstract}
Estimation of the fatigue life of pipelines under the random load was carried out. The power spectrum density of the stress on the dangerous location was calculated by the finite element method. The frequency method was implemented into the vibration fatigue life prediction. The applicability of the four models of the direct frequency method was analysis, which compare with the indirect frequency method. The results show that power spectrum density of the stress has the characteristic of two peaks. The probability density function of the stress amplitude calculated by different model have great differences. The application of the Dirlik model and ZB model get the reasonable value of the fatigue life. The fatigue life of the pipeline was optimized by changing the value of the fillet.
\end{abstract}

\section{Introduction}

The fatigue prediction under the random load is more difficult and complicate than under the conventional load with consistent amplitude, especially on the aspect of getting the response and the prediction model. (1) Express and getting the response. As the real load is random, it is difficult to express it in time domain. Power spectrum density can give well expression of it in frequency domain. The pipeline have great quantity and kinds of shapes, which makes its dynamic characteristic to be random and dynamic response to be difficult acquired. It have great significance to get the response based on the random load and dynamic characteristic. (2) Prediction model of vibration fatigue life. The main idea of this method is that calculate of the fatigue life is based on power spectral density [1-3]. The key step is to acquire the probability density function of the response. A lot of mode was advanced by many authors [4-9], but rarely can be found in the pipeline structure. Wang Lin [4] analysis the fatigue life of pressure piping system under random excitation, got the fatigue life with formula from power spectral density. Wang Shuai [8], from the view point of theoretical analysis and simulation, analysis dynamic strength properties of piping structural at a single, multi-axis random vibration loads and provides a quantitative assessment method of the strength. These studies established a general method of vibration fatigue life prediction, but is not fully suitable for power spectral density of stress with two peaks. And consider the characteristics of the structure and the load, the applicability of different models in the pipeline vibration fatigue analysis is also to be studied. The dangerous position and the power spectral density of stress were obtained by random vibration response analysis. Four models in the Frequency Domain analysis are evaluation. The results calculated from indirect method was the criterion. The results show that Dirlik model and ZB model get the reasonable value. Finally, vibration fatigue life is optimized by changing the radius which greatly improving the life of the pipeline.

\section{Power Spectrum Density of the Stress on the Dangerous Location}

The dynamic response of the structure is the foundation of fatigue life prediction analysis. It's time-consuming to get time domain response from finite element method, but easy to get frequency domain response. The main steps are including the establishment of the model, material and 
boundary condition, results and analysis.

Establishment of the Model. The research object of this paper is a pipeline of engine which mechanical environment is complex. One end of the tube is connected to the thrust chamber and the other end is connected to the oxygen turbo pump. The pipeline structure comprises a ball head, catheters, plug and triplet. The three-dimensional solid model built by Proe is shown in Fig. 1. In order to obtain the accurate dynamic response, Model of ball head was built by solid185 solid element. The fine mesh element was shown in .

Fig. 2. The number of elements is 111, 360. For the rest, beam188 elements were used. A Cartesian coordinate system is established in the triplet direction, the $\mathrm{X}$-axis direction consist to the ball, Y-axis direction consist to the plug, Z-axis direction consist to the catheter.

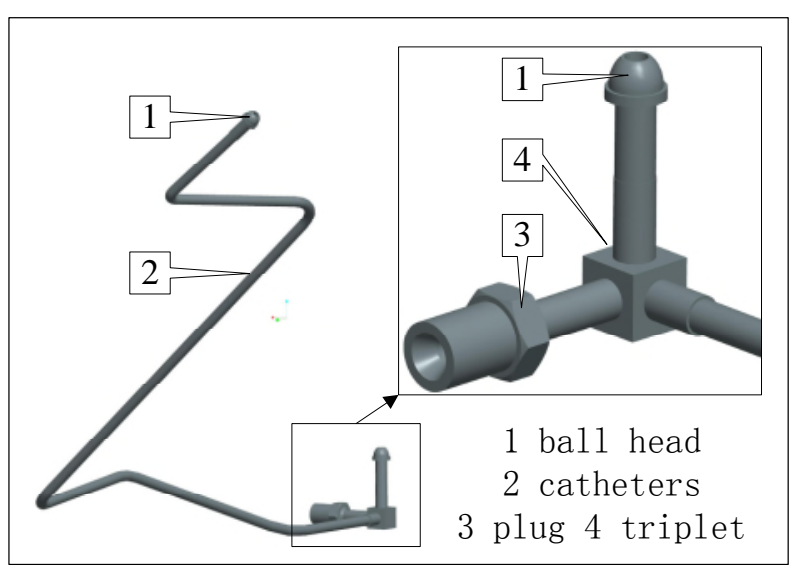

Fig. 1 Model of geometry.

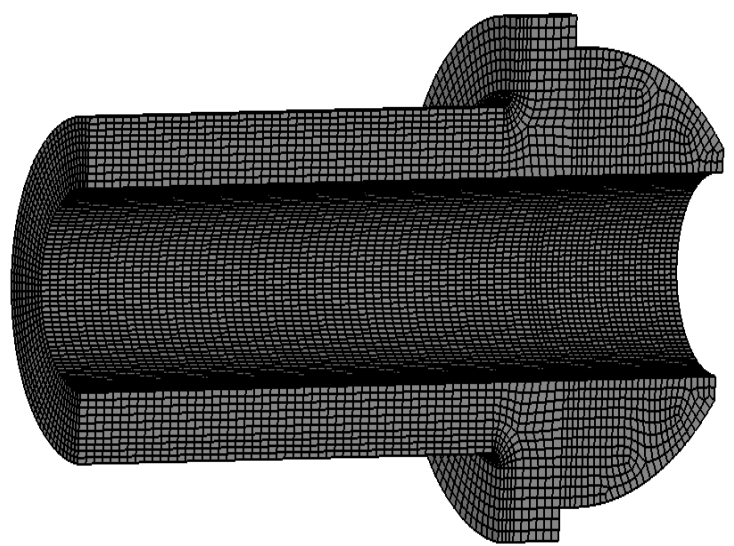

Fig. 2 Fine gridding of the bulb.

Material and Boundary Condition. The material parameters of $1 \mathrm{Cr} 18 \mathrm{Ni}$ Ti was shown in Table 1 . The relationship between the applied stress and fatigue life $\mathrm{N}$ called $\mathrm{S}-\mathrm{N}$ curve. The ultimate tensile strength is UTS. When $\mathrm{N}=1000$, the stress $\mathrm{S} 1=0.9 *$ UTS. When NC1 $=1 \mathrm{E} 6$, stress $\mathrm{S} 2=0.357$ * UTS. The slope of two lines is calculated as follows:

$$
b_{1}=\frac{\log (\mathrm{S} 2)-\log (\mathrm{S} 1)}{\log (\mathrm{NC} 1)-3} . \quad b_{2}=\frac{b_{1}}{2+b_{1}} .
$$

Table 1. Material property.

\begin{tabular}{cccccc}
\hline material & $E / \mathrm{MPa}$ & $\mu$ & $\rho /\left(\mathrm{Kg} / \mathrm{m}^{3}\right)$ & $\sigma_{s} / \mathrm{MPa}$ & $\sigma_{b} / \mathrm{MPa}$ \\
steel & $2.1 \mathrm{E} 5$ & 0.3 & $7.8 \mathrm{E} 3$ & 200 & 650 \\
\hline
\end{tabular}

The input load is power spectral density of measured acceleration as shown in Fig. 3 Power spectrum density of acceleration Fig. 3. The direction is the same with the coordinate axes. The constraints are fix complied with the actual installation condition of the ball head.

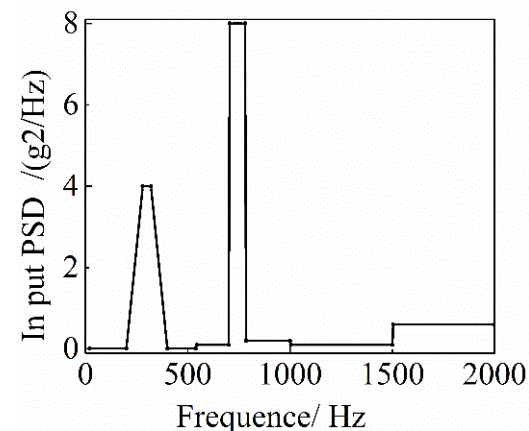

Fig. 3 Power spectrum density of acceleration.

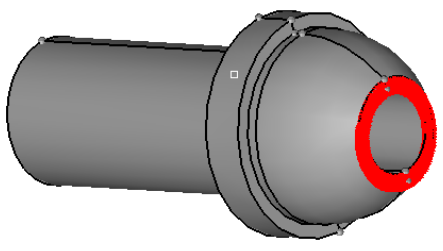

Fig. 4 Constrain of pipeline.

Calculate and Analysis of Dynamic Response. The dynamic characteristics and response of 
the structure was obtained by the finite element random vibration analysis. The modal frequency values were shown in Table 2. The displacement and stress response was shown in Table 3.

Table 2. Frequency of front six modes.

\begin{tabular}{ccccccc}
\hline step & 1 & 2 & 3 & 4 & 5 & 6 \\
\hline frequency $/ \mathrm{Hz}$ & 45 & 87 & 117 & 189 & 211 & 323 \\
\hline
\end{tabular}

Table 3. RMS of stress and displacement.

\begin{tabular}{ccccc}
\hline direction & $\sigma / \mathrm{Mpa}$ & location & $\mathrm{u} / \mathrm{mm}$ & location \\
\hline $\mathrm{X}$ & 36.6 & seal & 0.6 & middle \\
$\mathrm{Y}$ & 138.6 & $\begin{array}{c}\text { root of the } \\
\text { ball head }\end{array}$ & 0.3 & middle \\
$\mathrm{Z}$ & 31.1 & seal & 0.2 & middle \\
\hline
\end{tabular}

From the results, the dangerous locations are the root of the ball head when the load is $\mathrm{Y}$ direction. The power spectral density of stress was shown in Fig. 5. The frequency of the first peak of PSD cover the $5^{\text {th }}, 6^{\text {th }}$ modal frequency.

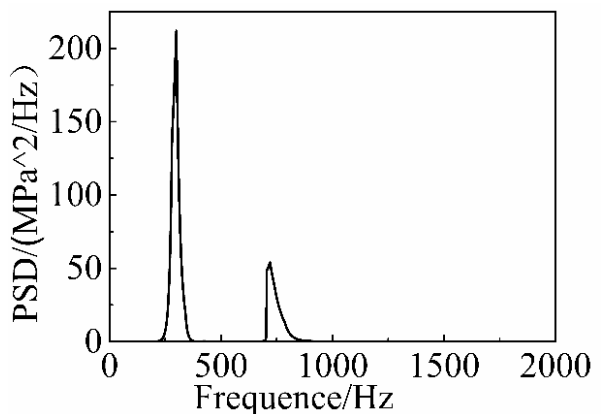

Fig. 5 Power spectrum density (PSD) of stress.

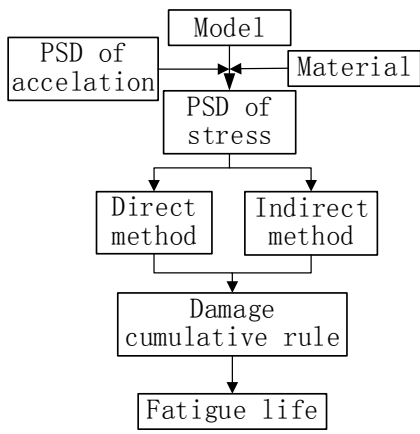

Fig. 6 Flow chart of vibration fatigue life analysis.

\section{Frequency Method of Vibration Fatigue Life Prediction on Pipeline}

Usually estimating structural vibration fatigue life from the power spectrum density of the stress is known as frequency-domain methods. The frequency method comprise the direct method and indirect method. The basic flow of vibration fatigue life analysis is shown in Fig. 6.

Direct Frequency Method. The amplitude probability density function of stress is determined by the spectral moment's parameters. However, there is no analytical solution exist [11]. The main models are narrow band model, narrowband correction model, Dirlik model, Zhao-Baker model.

(1) Narrow Band model, for case of the power spectral density of stress is narrowband, the probability density function of stress amplitude can be expressed by a Rayleigh distribution, the specific expression as follows:

$$
p_{\mathrm{a}}(\mathrm{s})=\frac{s}{\lambda_{0}} \exp \left(-\frac{\mathrm{s}^{2}}{2 \lambda_{0}}\right)
$$

where, $s$ is the stress amplitude, $\lambda_{0}$ is spectral moment's parameters of the power spectral density, specific expression is as follows:

$$
\lambda_{n}=\int_{0}^{\infty} \omega^{n} W(\omega) \mathrm{d} \omega
$$

where, $\omega$ is the frequency, is one sided power spectral density, $\mathrm{n}$ ranging from 0 to 4 . 
The fatigue damage expression under Narrowband power spectral density is as follows:

$$
\overline{D_{N B}}=v_{p} C^{-1} \int_{0}^{+\infty} s^{k} p_{\mathrm{a}}(\mathrm{s}) \mathrm{ds} \text {. }
$$

where, $\mathrm{s}$ is the stress amplitude, $v_{p}$ is the peak rate of the random process, $\mathrm{C}$ and $\mathrm{k}$ are fatigue parameters of material.

(2) Narrowband correction model, The Narrowband model not adapt to power spectral density with broadband [12]. The correction model have been proposed by Wirsching and Light [13]. The specific expression is as follows:

$$
\bar{D}_{\mathrm{wL}}=\lambda_{W L} \bar{D}_{N B} \text {. }
$$

where, $\lambda_{w L}$ is the empirical coefficient, it is determined by the material parameters and bandwidth parameters.

Lutes LD [10] proposed another correction model as formula (6).

$\bar{D}_{L D}^{0.75}=\alpha_{0.75}^{2} \bar{D}_{N B}$.

where, $\alpha_{0.75}^{2}$ is the correction factor, it is determined by bandwidth parameters.

Tovo-Benasciutti [11] proposes a modified model by a linear combination of narrow band .

$\bar{D}_{\text {тв }}=\lambda_{T B} \bar{D}_{N B}$.

where, $\lambda_{T B}$ is the correction factor.

(3) Dirlik model, Dirlik [14] proposes a model (8) by Monte Carlo techniques, which is fit for broadband PSD.

$$
p_{a}(\mathrm{~s})=\frac{1}{\sigma_{X}}\left[\frac{D_{1}}{Q} \mathrm{e}^{-\frac{Z}{Q}}+\frac{D_{2} Z}{R^{2}} \mathrm{e}^{-\frac{Z^{2}}{2 R^{2}}}+D_{3} \mathrm{Ze}^{-\frac{Z^{2}}{2}}\right] .
$$

$\mathrm{Z}$ is normalized stress. The other parameters are express by moments parameters of PSD.

(4) Zhao-Baker model, Zhao-Baker [15] consider that the probability density function of stress amplitude is a linear combination of Rayleigh and Weibull distribution, as specified in the formula (9).

$$
p_{a}(\mathrm{~s})=\mathrm{W} \alpha \beta \mathrm{s}^{\beta-1} e^{-\alpha s^{\beta}}+(1-\mathrm{w}) \mathrm{se}^{-\frac{s^{2}}{2}} .
$$

where, $\mathrm{w}$ is a weighting coefficient, which ranges from 0 to $1 . \alpha, \beta$ is the Weibull parameters.

According to the linear cumulative damage rule, the fatigue damage is as follows.

$$
\mathrm{D}=v_{a} C^{-1} \int_{0}^{+\infty} s^{k} p_{a}(\mathrm{~s}) \mathrm{d} \mathrm{s}
$$

where, $v_{a}$ is a level crossing rate, $\mathrm{C}$ is a material parameter.The fatigue life obtained by inverse fatigue damage:

$$
\mathrm{T}=\frac{1}{D}
$$

\section{Indirect Frequency Method}

The triangular series is used to translate PSD from frequency domain to time domain.

$$
q(t)=\sum_{k=1}^{n} a_{k} \sin \left(2 \pi f_{k} t+\alpha_{k}\right)
$$

$\alpha_{k}$ is the phase with value within $(0,2 \pi), a_{k}$ is amplitude, $f_{k}$ is frequency.

Through harmonic superposition method, the domain samples convert from the power spectral density shown in Fig. 7. 


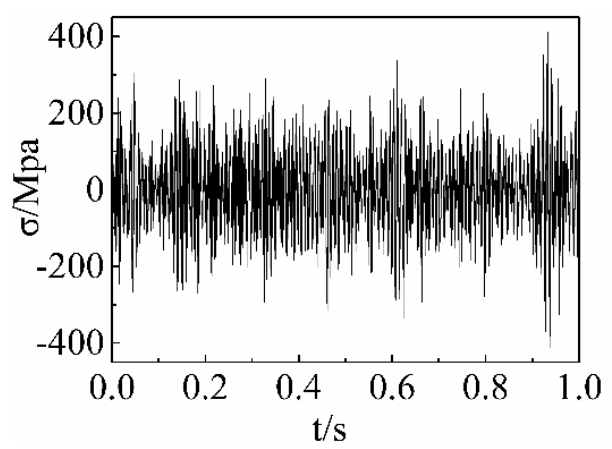

Fig. 7 Swatch of stress in time field.

\section{The Result and Analysis of Vibration Fatigue Life Prediction on Pipeline}

Results of Direct Frequency Method. The fatigue life is calculated by matlab and design life. The particular result as follows. The fatigue life of the pipeline at a danger point is shown in Table 4 . The result showed that, the fatigue life calculated by NB model is the shortest. From the probability density function (Fig. 8) of stress amplitude, the value calculated from Dirlik and ZB model is greater than NB model at low stress, but reverse at high stress.

Table 4. Value of fatigue life.

\begin{tabular}{|c|c|c|c|c|c|c|c|}
\hline $\begin{array}{c}\text { Calculate } \\
\text { model }\end{array}$ & NB & WL & LD & TB & Dirlik & ZB & Time \\
\hline $\mathrm{T} / \mathrm{S}$ & $3.0984 \mathrm{e}+03$ & $4.6409 e+03$ & $3.5767 \mathrm{e}+03$ & $3.9047 \mathrm{e}+03$ & $3.7221 \mathrm{e}+03$ & $3.7627 \mathrm{e}+03$ & $3.669 \mathrm{e}+3$ \\
\hline
\end{tabular}

Results of Indirect Frequency Method. In order to ensure the reasonableness of the time domain signal, The power spectral density of stress is calculate from time domain signal to compare with original power spectrum density curve as in Fig. 8 Probability density of stress amplitude. Fig. 9. The figure shows two curves substantially coincide, a time domain signal is preferably simulate the original stress power spectral density. Connect fatigue characteristics of material (S-N curve) and linear cumulative damage rule, the average fatigue life calculate by time sample is 3669 s. The results show Dirlik model and ZB model is more suitable for the fatigue life prediction.

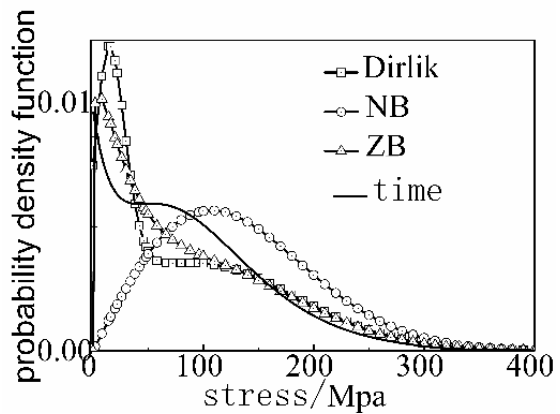

Fig. 8 Probability density of stress amplitude.

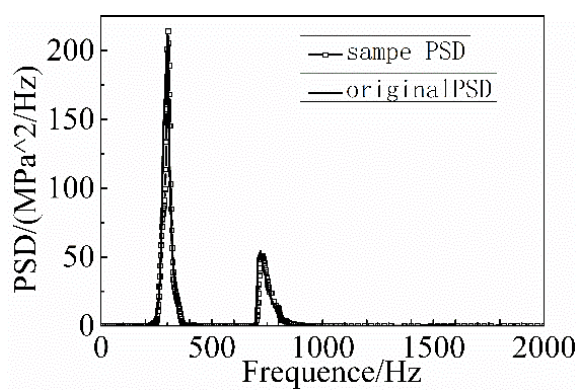

Fig. 9 Contrast of PSD.

\section{Optimization of Vibration Fatigue Life of Pipeline}

The fatigue life of the pipeline is optimized by changing the ball radius $\mathrm{R}$ such as Fig. 10. The PSD of stress is show as Fig. 10 Radius of corner.

Fig. 11 PSD of stress. Because the change does not affect the dynamic characteristics of the entire pipeline, so the dynamic response frequency corresponding to the peak do not change but amplitude with increasing radius decreases gradually. The fatigue life with different radius is Calculate by matlab programming as shown in Table 5. The fatigue life have greater improvement than the previous. The increase proportion is $141.4 \%, 332.85,541.2 \%$ by different radius ratio. 


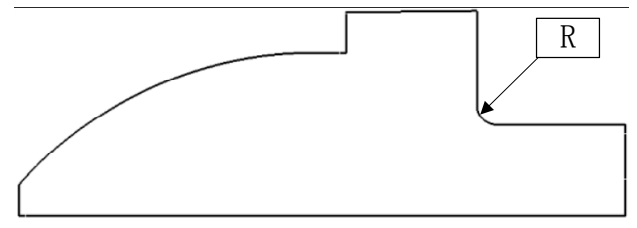

Fig. 10 Radius of corner.

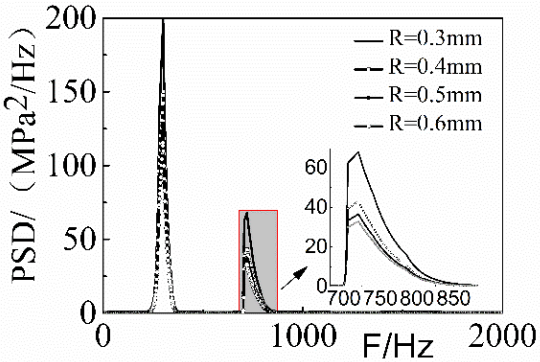

Fig. 11 PSD of stress.

Table 5. Value of fatigue life.

\begin{tabular}{ccc}
\hline \multirow{2}{*}{$\mathrm{R} / \mathrm{mm}$} & \multicolumn{2}{c}{$\mathrm{T} / \mathrm{S}$} \\
\cline { 2 - 3 } & Dirlik & $\mathrm{ZB}$ \\
\hline 0.4 & $8.986 \mathrm{e}+03$ & $9.487 \mathrm{e}+03$ \\
0.5 & $1.611 \mathrm{e}+04$ & $1.699 \mathrm{e}+04$ \\
0.6 & $2.386 \mathrm{e}+04$ & $2.515 \mathrm{e}+04$ \\
\hline
\end{tabular}

\section{Conclusion}

Vibration Fatigue Life Prediction for pipeline was carried out by this paper. The high-precision finite element model was built. The stress PSD of dangerous point was calculate by finite element analysis. The probability density distribution model of stress amplitude was determined. The fatigue life was got by frequency method and fatigue life optimization was realized by changing the corner radius. The conclusions are as follow. (1) The root of ball fillet is the dangerous position of the pipeline. Its power spectral density of stress have a bimodal characteristics and the first peak coincide with the five or six natural frequencies. (2) Dirlik model, ZB model have great applicability on fatigue life calculation (3) The fatigue life is improved by changing the ball radius R.

\section{References}

[1] M. Z. Wang, W. X. Yao, Frequency domain method for fatigue life analysis on notched specimens under random vibration loading, J. Nanjing University Aeronaut. Astronaut. 40(4) (2008) 489-492.

[2] C. R. Meng, B. Y. Lu, Estimation on vibration fatigue life-span under random loading based on PSD, J. Mach. Des. 5(26) (2009) 73-75.

[3] L. C. Gu, K. Wang, Structure random fatigue life simulation based on power spectral density, Comp. Modern. 2(174) (2010) 143-146.

[4] L. Wang, Q. Ni, Q. Zhang, et al, Estimation of fatigue life of pressure piping system under random excitations, J. Huazhong Univ. Sci. Tech. (Nature Science Edition), 31(12) (2003) 100-110.

[5] C. Li, An approach based on power spectral density for fatigue life estimation, Mach. Des. Res. 4(21) (2003) 6-8.

[6] D. Benasciutti, R. Tovo, Cycle distribution and fatigue damage assessment in broad-band non-Gaussian random processes, Prob. Eng. Mech. 20 (2005) 115-127.

[7] H. Andrew. A frequency domain approach for fatigue life estimation from finite element analysis, In: International conference on damage assessment of structure, 1999.

[8] S. Wang, B. L. Li, L. Jia, The fatigue damage research of space pipeline structures under uniaxial/multiaxial random vibration, Struct. Envir. Eng. 39(6) (2012) 36-41. 
[9] M. Paulus, A. Dasgupta, Ed. Habtour, Life estimation model of a cantilevered beam subjected to complex random vibration, Fatigue Fract. Eng. Mater. Struct. 35 (2012) 1058-1070.

[10]L. D. Lutes, S. Sarkani, Stochastic analysis of structural and mechanical vibrations. Prentice-Hall, 1997.

[11]D. Benasciutti, R. Tovo, Comparison of spectral methods for fatigue analysis of broad-band Gaussian random processes, Prob. Eng. Mech. 21 (2006) 287-299.

[12]I. Rychlik, On the 'narrow-band' approximation for expected fatigue damage. Prob. Eng. Mech. 8 (1993) 1-4.

[13]P. H. Wirsching, C. L. Light, Fatigue under wide band random stresses. J. Struct. Div. ASCE, 106(7) (1980) 1593-1607.

[14]T. Dirlik. Application of computers in fatigue analysis. PhD thesis. UK: University of Warwick, 1985.

[15]B. Zhao, On the probability density function of rainflow stress range for stationary Gaussian processes. Int. J. Fatigue, 14(2) (1992) 121-135. 